\title{
Operando Transmission Electron Microscopy of Catalyst Decoking Activated by Ultraviolet Surface Plasmons
}

\author{
Wei-Chang Yang ${ }^{1}$, Canhui Wang ${ }^{1}$, David Raciti ${ }^{1}$, Amit Agrawal $^{1}$, Ronald Marx ${ }^{2}$ and Renu Sharma ${ }^{1}$ \\ ${ }^{1}$ National Institute of Standards and Technology, Gaithersburg, Maryland, United States, ${ }^{2}$ DENSsolutions, \\ Delft, Zuid-Holland, Netherlands
}

Deactivation of catalysts by carbon deposition (coking) is a persisting concern in industrial catalytic processes and costs billions routinely for catalyst replacements and production interruptions [1]. The cause of coking involves adsorption of various types of carbon species and their decomposition into carbon on the surface of transition metal catalysts, such as $\mathrm{Ni}, \mathrm{Co}$, and $\mathrm{Fe}$, during a large number of petrochemicals processes $[1,2]$. Activating a carbon-consuming reaction, such as reverse Boudouard reaction $(\mathrm{RBR}): \mathrm{C}_{(s)}$ $+\mathrm{CO}_{2(g)} \rightarrow 2 \mathrm{CO}_{(g)}$, presumably gasify the deposited carbon and potentially reactivates the catalytic surfaces. However, the activation energy of RBR, ranging from $2.91 \mathrm{eV}$ to $3.9 \mathrm{eV}$, demands for a hightemperature environment beyond $600{ }^{\circ} \mathrm{C}$ that could lead to the further loss of catalytic activity by sintering metallic nanoparticles.

Herein, we employed environmental transmission electron microscopy (ETEM) and a direct electron detector to show that the graphitic carbon structures on the Ni catalyst surface were removed by RBR at room temperature in a $\mathrm{CO}_{2}$ environment when coupling an $80-\mathrm{keV}$ electron beam with ultraviolet (UV) LSP modes of the adjacent $\mathrm{Al}-\mathrm{Al}_{2} \mathrm{O}_{3}$ core-shell nanoparticles $(100 \pm 26 \mathrm{~nm}$ in diameter), referred as $\mathrm{Al}$ nanoparticles hereafter. In-situ low-loss electron energy-loss spectroscopy (EELS) [3] and the metallic nanoparticles boundary element method (MNPBEM) simulations [4] confirmed that the LSP-induced RBR only took place near the Al nanoparticle surface, where the field enhancement of the UV mode was located. We further performed operando TEM measurements of the room-temperature RBR using a mixture of graphite flakes and Al nanoparticles in a gas-cell TEM holder, and monitored the gas composition of the exhaust using gas chromatography-mass spectrometry (GC-MS), attempting to bridge the surface reaction mechanism to the $\mathrm{CO}$ production rate.

High-resolution transmission electron microscopy (HRTEM) images were collected using an electron flux of $0.28 \mathrm{~A} / \mathrm{cm}^{2}$ to show that $\mathrm{Ni}$ catalysts were enclosed by graphitic carbon and multiwalled carbon nanotubes (MWCNTs) in Fig. 1a. The added Al nanoparticles were usually found attached to the graphitic carbon and MWCNTs (Fig. 1b). As the electron flux was increased to eight times higher than the typical imaging condition in a $\mathrm{CO}_{2}$ environment, the layers of graphite (with a spacing of $\approx 0.34 \mathrm{~nm}$ ) were removed at a rate of $\approx 1.3$ layers per second, starting from the layer of graphite in contact with the $\mathrm{Al}$ nanoparticle surface. After 15 seconds of reaction, the Ni catalyst was attached to the Al nanoparticle surface (Fig. 1c), showing no trace of the layered graphitic structures. To measure the CO production of $\mathrm{RBR}$ and correlate it to the etching of graphite, we mixed the Al nanoparticles with graphite flakes on the silicon nitride chip of a gas cell holder. Each window ( $3 \mu \mathrm{m}$ in diameter) was covered by the graphite flakes and their thickness was measured using energy-filter TEM (EFTEM) in Fig. 1d. We then normalized the as-measured thickness with the theoretical inelastic electron scattering mean free path of graphite, as shown in the color scale of Fig. $1 \mathrm{~d}$ and e (light colors indicating thick areas) [5]. After exciting the LSP modes on $\mathrm{Al}$ nanoparticles in $\mathrm{CO}_{2}$ environment for $30 \mathrm{~min}$ using the parallel electron beam, the graphite was etched (Fig. 1e). In the meantime, the produced $\mathrm{CO}$ was accumulated in the gas cell holder for a detectable quantity and batch injected into the GC-MS after multiple windows of graphite flakes 
were etched under the same condition. The $\mathrm{CO}$ to $\mathrm{CO}_{2}$ concentration ratio was displayed as a function of etched graphite volume in Fig. 1f. This curve could be extrapolated to estimate the CO production rate at a small graphite volume and compared with the graphitic carbon etching rate modulated by the plasmonic field enhancement (Fig. 1 from b to c). Our approach shows the potential of operando TEM measurements that correlate the nanoscale graphite-etching mechanism to the overall reaction rate, and the importance of adopting GC-MS for its high sensitivity and selectivity to measure the gas composition altered by an LSP-inducted reaction. The knowledge gained from operando TEM will provide nanoscale design rules to develop light-coupled plasmonic nanostructures for catalyst reactivation at the industrial level.
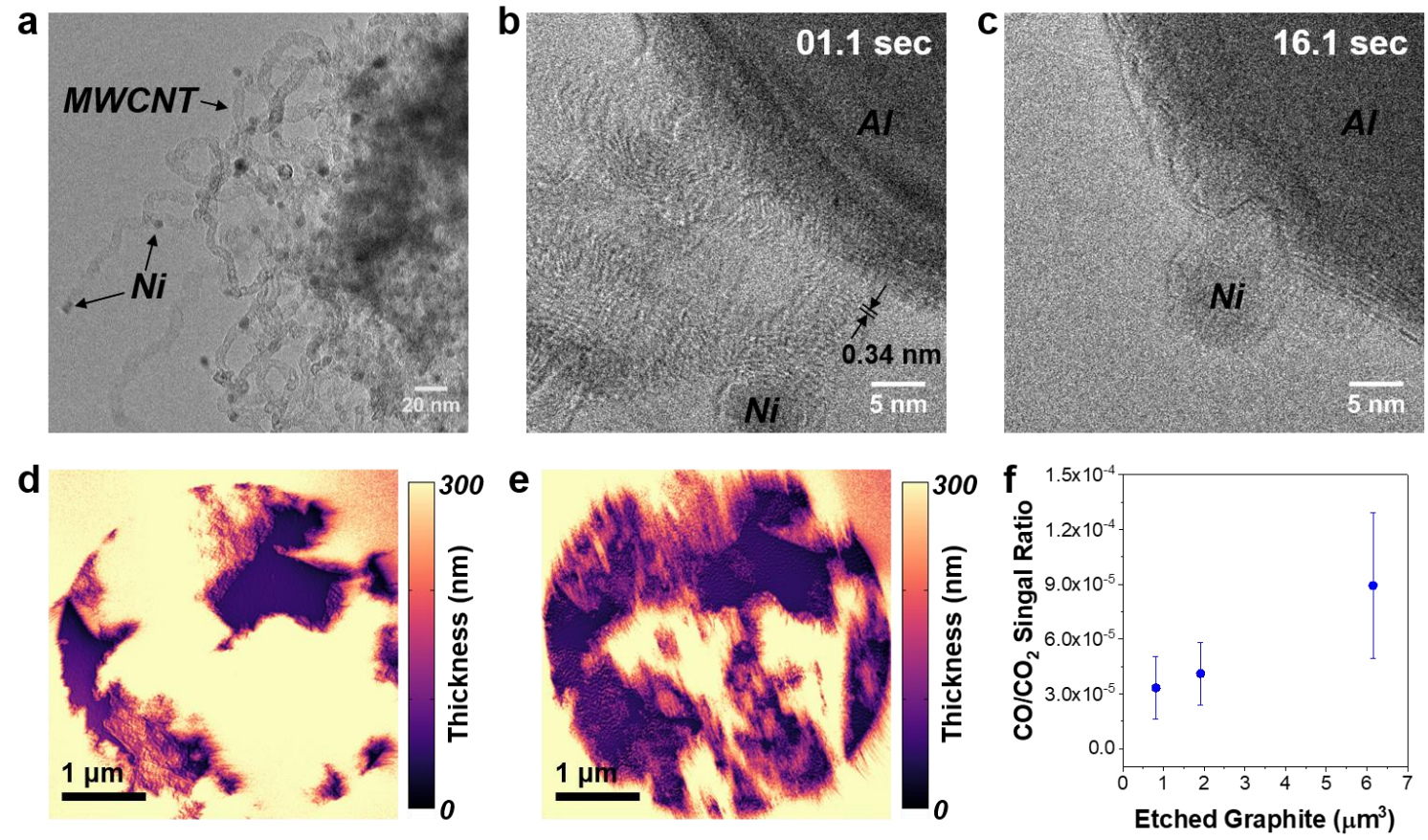

Figure 1. a, TEM image of Ni catalysts covered by graphitic carbon and MWCNTs. b, HRTEM image revealing layered graphitic carbon structures in contact with the Al nanoparticles. c, HRTEM of a $\mathrm{Ni}$ catalyst after LSP-induced RBR showing no graphic carbon on the Ni surface. d, Thickness distribution, measured using EFTEM, of graphite flakes that were mixed with Al nanoparticles and covering a throughhole on a Si3N4 substrate. e, EFTEM thickness map displaying a reduced graphite thickness due to carbon consumption during the LSP-induced RBR. f, CO-to-CO2 signal ratio measured by GC-MS displayed as a function of total graphite volume that was etched during the LSP-induced RBR. The uncertainty is determined by the single standard deviation of $\mathrm{CO}$ signal fitting, using a Gaussian profile, normalized by the $\mathrm{CO} 2$ signal.

\section{References}

[1] P. Forzatti and L. Lietti, Catalysis Today 52 (1999), p. 165.

[2] M. D. Argyle and C. H. Bartholomew, Catalysts 5 (2015), p. 145.

[3] W.-C. D Yang et al., Nature Materials 18 (2019), p. 614.

[4] U. Hohenester, Computer Physics Communications 185 (2014), p. 1177.

[5] S. Tanuma, C. J. Powell, and D. R. Penn, Surf. Interf. Anal. 21, (1993), p. 165. 